\title{
Visuo-Haptic Discrimination of Viscoelastic Materials
}

\author{
Ozan Caldiran, Student Member, IEEE, Hong Z. Tan, Fellow, IEEE, \\ and Cagatay Basdogan, Senior Member, IEEE
}

\begin{abstract}
In our daily lives, we interact with different types of deformable materials. Regarding their mechanical behavior, some of those materials lie in a range that is between purely elastic and purely viscous. This range of mechanical behavior is described as viscoelasticity. In certain types of haptic interactions such as assessment of ripeness of fruit, firmness of cheese, and consistency of organ tissue, we rely heavily on our haptic perception of viscoelastic materials. The relationship between the mechanical behavior of viscoelastic materials and our perception of them has been investigated in the field of psychorheology. However, our knowledge on how we perceive viscoelastic materials is still quite limited though some research work has already been done on purely elastic and purely viscous materials. History- and frequency-dependent behavior of viscoelastic materials result in a complex time-dependent response, which requires relatively more sophisticated models to investigate their behavior than those of purely elastic and viscous materials. In this study, we model viscoelasticity using a "springpot" (i.e., fractional order derivative element) and express its behavior in the frequency domain using two physical parameters: "magnitude" and "phase" of complex stiffness. In the frequency domain, we are able to devise signal detection experiments where we can investigate the perception of viscoelastic materials using the perceptual terms of "firmness" and "bounciness", corresponding to the physical parameters of "magnitude" and "phase". The results of our experiments show that the JND for bounciness increases linearly with increasing "phase", following Weber's law, while the JND for firmness is surprisingly independent of the level of "phase".
\end{abstract}

Index Terms-Viscoelasticity, psychorheology, visuo-haptic perception, bounciness, firmness, softness, hardness, perception, psychophysical experiments, springpot.

\section{INTRODUCTION}

$\mathrm{V}$ ISCOELASTIC material behavior is commonly observed in nature especially in biological materials [1]. In interaction scenarios such as squeezing a piece of fruit to assess its ripeness or palpating soft organ tissues to assess their consistency, we have to rely on our haptic perception of viscoelasticity. In certain settings, our judgments about the viscoelastic properties of these materials can be of critical importance. For example, tissue consistency is one of the four criteria (the $4 \mathrm{Cs}$ ) used by surgeons to assess muscle debridement alongside color, contractility, and capacity to bleed; though research shows that decisions made based on the $4 \mathrm{Cs}$ do not agree with histopathological findings [2]. Investigating the discrimination thresholds relating to our haptic perception of material consistency will help us to gain insight into the reliability of our judgments in such critical settings.

Besides, the design of medical simulation and teleoperation systems can also benefit from the knowledge of the perceptual thresholds. Interactive medical simulations require real-time computation of force response of a modeled tissue. However, developers may not be able to fulfill this requirement unless they make a sacrifice in the fidelity of the models [3]. In this regard, our perceptual thresholds can

- O. Caldiran and C. Basdogan are with the Department of Mechanical Engineering, Koc University, Istanbul 34450, Turkey.

E-mail: \{ocaldiran13, cbasdogan\}@ku.edu.tr

- H. Z. Tan is with the Haptic Interface Research Laboratory, School of Electrical and Computer Engineering at Purdue University, West Lafayette, IN, 47907, USA.

E-mail: hongtan@purdue.edu.

Manuscript received XX XX, 2018; revised XX XX, 2019 be used as a guideline for determining this fidelity. Similarly, teleoperation systems require real-time transmission of data between local and remote sites. One approach to reducing data transfer and achieve real-time interaction is to exploit the limitations of the human perceptual system. Researchers have proposed data reduction techniques based on the justnoticeable differences (JNDs) relevant to haptic modality [4], [5]. These techniques can be tailored to the medical domain, or any other domain relating to viscoelastic materials, using a systematic knowledge on the thresholds of haptic perception of viscoelasticity.

However, most studies on haptic perception of deformable materials have focused on either elasticity or viscosity, but not both [6]. Researchers scrutinized the question of how humans judge the stiffness of a spring or the viscosity of a liquid. They have investigated the prevalent haptic cues used in stiffness or viscosity judgment [7], [8], [9], [10], [11]; integration of information coming from different cues or senses [8], [12]; and effects of time delay in force feedback [13], [14]; exploration strategies [15], [16], tool use [9], [15], [17]; boundary crossings [10], [11]; the relation of perception and action, and effects of arm posture and force direction [18], [19]. Perception studies on elasticity and viscosity have also determined the respective JNDs under different conditions (see Table $1^{1}$ for JNDs reported in earlier studies as Weber fractions $(\mathrm{WF})^{2}$ ).

1. The table includes results from exemplar studies that might not be fully representative.

2. Weber fraction is the ratio of JND to the reference stimulus intensity. Although Weber fraction is a convenient way of comparing JNDs, it may not always hold [20]. 
TABLE 1

Elasticity and Viscosity JND as Weber Fractions (WF)

\begin{tabular}{|c|c|c|c|c|c|}
\hline Effect of & & Pub. & Stimulus & Feedback & $\mathrm{WF}$ \\
\hline \multirow{4}{*}{\multicolumn{2}{|c|}{$\begin{array}{l}\text { Visual } \\
\text { feedback }\end{array}$}} & {$[21]$} & Elastic & $\begin{array}{l}\mathrm{K} \\
\mathrm{K}+\mathrm{V}\end{array}$ & $\begin{array}{l}6-8 \% \\
5 \% \\
\end{array}$ \\
\hline & & {$[22]$} & Elastic & $\begin{array}{l}\mathrm{K} \\
\mathrm{K}+\mathrm{V}\end{array}$ & $\begin{array}{l}14.2 \% \\
17.2 \%\end{array}$ \\
\hline & & \multirow{2}{*}{ [23] } & $\begin{array}{l}\text { Elastic } \\
\text { (Hard) }\end{array}$ & $\begin{array}{l}\mathrm{H} \\
\mathrm{H}+\mathrm{V} \\
\mathrm{V}\end{array}$ & $\begin{array}{l}18 \% \\
23 \% \\
200 \%\end{array}$ \\
\hline & & & $\begin{array}{l}\text { Elastic } \\
\text { (Soft) }\end{array}$ & $\begin{array}{l}\mathrm{H} \\
\mathrm{H}+\mathrm{V} \\
\mathrm{V}\end{array}$ & $\begin{array}{l}16 \% \\
15 \% \\
38 \%\end{array}$ \\
\hline \multicolumn{2}{|l|}{$\begin{array}{l}\text { Cutaneous } \\
\text { feedback }\end{array}$} & {$[8]$} & Elastic & $\begin{array}{l}\mathrm{H} \\
\mathrm{K}\end{array}$ & $\begin{array}{l}15 \% \\
50 \% \\
\end{array}$ \\
\hline \multicolumn{2}{|l|}{$\begin{array}{l}\text { Material } \\
\text { type }\end{array}$} & $\begin{array}{l}{[24]} \\
{[25]} \\
{[26]} \\
{[27]} \\
\end{array}$ & $\begin{array}{l}\text { Elastic } \\
\text { Viscous } \\
\text { Elastic } \\
\text { Viscous } \\
\end{array}$ & $\begin{array}{l}\mathrm{K} \\
\mathrm{K} \\
\mathrm{K} \\
\mathrm{K} \\
\end{array}$ & $\begin{array}{l}8 \% \\
13.6 \% \\
23 \% \\
34 \% \\
\end{array}$ \\
\hline Displacement & $\begin{array}{l}\text { Fixed } \\
\text { Roving }\end{array}$ & {$[24]$} & Elastic & $\begin{array}{l}\mathrm{K} \\
\mathrm{K} \\
\end{array}$ & $\begin{array}{l}8 \% \\
22 \% \\
\end{array}$ \\
\hline Feedback: & K: Kinesthe & : Visu & A: Haptic (Kinestl & ic and Tactile) & \\
\hline
\end{tabular}

Compared to viscoelasticity, both elasticity and viscosity are relatively straightforward to investigate since they are governed by constant ratios of the force response to displacement (elasticity) or to velocity (viscosity). On the other hand, viscoelasticity is a highly complex, history- and frequency-dependent material behavior (see Section 2). This complexity is also reflected in the mathematical formulation of the models and the increase in the number of modeling parameters. One possible approach to tackle these challenges is to use simplified models that can partially mimic the behavior of viscoelastic materials. In [28], a viscoelastic soft tissue model was simplified as a spring with time-delay in the response, and a nonlinear boundary contact layer. Nonetheless, such an approach results in a loss of fidelity in modeling and is not suitable for our purposes.

Previously, we have proposed to investigate viscoelastic materials in the frequency domain for psychophysical evaluation without any sacrifice in modeling [29]. Such a representation enables us to alter only a single physical quantity, magnitude or phase difference through the back transformation to the parameter space, irrespective of the chosen viscoelastic model. Utilizing this approach, we had investigated points of subjective equality between a Maxwell arm (a spring and a damper in series) and purely elastic and viscous models. Similar approaches have also been utilized before for investigating the haptic perception of dynamic systems comprised of mass, spring, and damper [14], [30], [31], [32]. Although these approaches require participants to follow a prescribed sinusoidal motion, they allow studying dynamic behavior that is otherwise not achieved so far according to the best of our knowledge. Moreover, such sinusoidal movement patterns can be viewed as application and release of pressure applied to deformable materials for assessing their softness [33], and repeating such loadingunloading cycles increases the sensory information gathered this way [34], [35], [36], [37], [38].

Although our earlier approach [29] made the psychophysical evaluation of viscoelastic material behavior possible, we have not utilized any perceptual terms to assess the subjective feelings of participants. Fortunately, in psychorheology, a branch of psychophysics that specializes in materials that flow such as viscous and viscoelastic materials, this issue has already been addressed [39]. In the psychorheology field, researchers have proposed lists of adjectives to describe the perceptual properties of rheological materials [40], [41], [42], [43]. They also tried to establish links between the physical aspects of the models and the adjectives through descriptive analysis [43], [44]. These links were then used to measure quality, acceptance or discriminability of certain products concerning the haptic sensory experience of consumers, especially in food and cosmetics research [45]. For example, researchers studied the effects of the fat content of dairy products [46], aging of cheese [47], or different brands of body lotions [48] on our perception.

In one of the earliest works of the psychorheology field, Scott-Blair and Coppen defined the "firmness" for a ball of California bitumen, which is a viscous material, as well as an elastic rubber specimen using a unified formulation [49]. The term "firmness" was chosen over "hardness" because the latter has different meanings in other branches of science [40], [41], [42]. Moreover, Scott-Blair and Coppen's realizations about the temporal characteristics of viscoelastic materials also led to the introduction of a new rheological model called springpot (Scott-Blair) element [50], [51], [52]. For a springpot element, the relationship between force $f$ and displacement $y$ is formulated as

$$
f=\chi D_{t}^{\alpha} y,
$$

where $D_{t}^{\alpha}=\left(d^{\alpha}\right) /\left(d t^{\alpha}\right)$ is the fractional derivative operator of order $\alpha$ with respect to time $t$, and $\chi$ is a quasi-property or coefficient whose units change with the derivative order.

Fractional derivatives can be thought of as a generalization of the more familiar concept of integer order derivatives to fractional number orders. For example, the fractional derivative of order one with respect to time applied to displacement $y\left(D_{t}^{1} y\right)$ is still the first derivative of $y$, i.e., velocity $\dot{y}$. So, a springpot element with $\alpha=0$ corresponds to a spring, and $\alpha=1$ corresponds to a damper. Moreover, if we analyze the force response of a springpot element in the frequency domain, we can almost decouple the contributions of the variables $\chi$ and $\alpha$ [53]. As depicted in Fig. $1, \chi$ scales the magnitude of the force response, whereas $\alpha$ determines the phase difference $\phi$ between the force and displacement. It should be noted that $\alpha$ also affects the force magnitude through the excitation frequency $\omega$. Further mathematical details are available in Section 2.

Under the light of the discussion above, we can view viscoelasticity as a spectrum of material behavior spanned from pure elasticity to pure viscosity. Hence, investigating JND of viscoelastic materials can provide us with insights about how JND varies along this spectrum since the earlier studies focused on its lower (elasticity) and upper (viscosity) bounds only. Tan et al. measured the compliance (reciprocal of stiffness) JND with a signal detection experiment [24], whereas Beauregard used the same method to determine viscosity JND [25]. Also, Jones and Hunter investigated both stiffness and viscosity JNDs with contralateral limbmatching experiments [26], [27]. In these studies, the Weber 


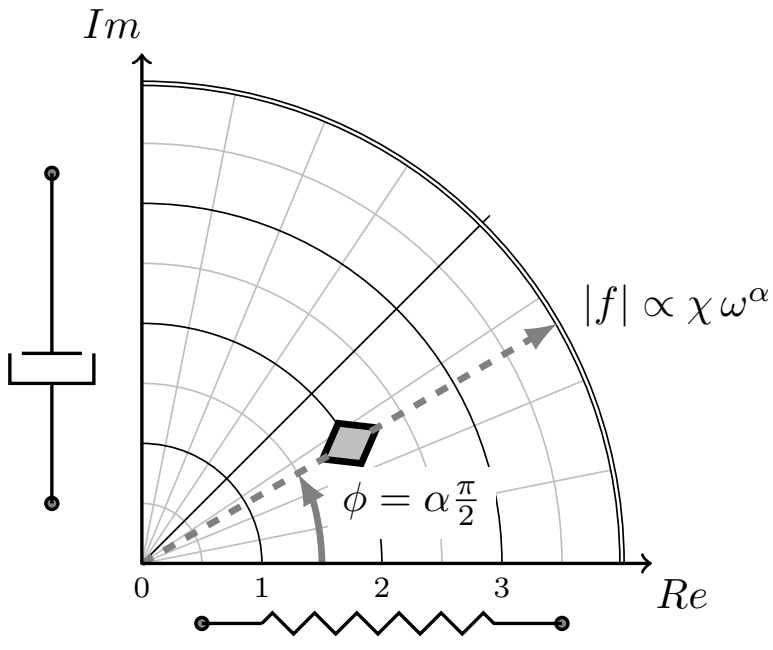

Fig. 1. Springpot element in polar coordinates. The angular coordinate describes the phase difference $\phi$ between force and displacement, while the radial coordinate describes the magnitude of the force response. For purely real (imaginary) coordinates, a springpot is equivalent to a spring (damper).

fractions (WFs) reported for viscosity $(13.6 \%$ in [25] and $34 \%$ in [27]) were higher than the WFs for elasticity ( $8 \%$ in [24] and $23 \%$ in [26]).

In the present study, we left the confines of the viscoelastic spectrum and probed further into it regarding the discrimination thresholds of "bounciness" and "firmness". Both terms were adopted from the psychorheology field. The terms "bounciness" and "firmness" describe the perceived phase difference and magnitude of a viscoelastic material response under displacement, respectively. In the next section, more detailed descriptions of these terms are given after an overview of viscoelastic material behavior in general, and springpot element in particular.

For psychophysical evaluations, we designed two signal detection experiments and measured the JNDs of perceived "firmness" and "bounciness" of the springpot element. In the experimental design, we used the frequency-domain approach introduced in our earlier study [29]. In the bounciness experiment, in line with earlier studies on the perception of dynamic systems, we visually displayed reference movement trajectories with a constant frequency. Thus, visual feedback was always present in our experiments. In that sense, our study falls in the category of visuo-haptic perception. Also, the stimuli were haptically rendered by a point-contact electromechanical device which allows us to tune the viscoelastic model parameters freely at the expense of reduced tactile information.

\section{BACKGROUND ON VISCOELASTICITY}

The mechanical response of viscoelastic materials is both time- and frequency-dependent. Viscoelastic materials can show both energy storage and loss characteristics depending on the loading frequency and history. Stress-relaxation and creep are two phenomena where we observe these two characteristics. Moreover, a phase difference between force and displacement occurs for a viscoelastic material under dynamic loading.
We use rheological models to represent these characteristics mathematically. Rheological models are specific configurations of spring, dashpot, and springpot elements. Integer derivative orders of 0 and 1 describe the forcedisplacement relationships of spring and dashpot (damper) elements, respectively. However, we require fractional order derivatives to model the same relationship in the case of springpot elements. Despite the computational complexity of the fractional order differentiation, rheological models utilizing a springpot element introduces several advantages over the ones composed of springs and dashpots alone (see applications in tissue modeling in [54], [55], [56], for example).

In the rest of this section, we first provide brief explanations of the common characteristics of viscoelastic materials and then the perceptual aspects of viscoelasticity based on the springpot element.

\subsection{Stress-Relaxation}

If a viscoelastic material is compressed at a sufficiently high rate and then held at the final displacement (ramp and hold input), we can observe a relaxation of the force response. Initially, a portion of the strain energy is stored, similar to an elastic material, but then some portion of this energy is dissipated (see Fig. 2a). In a quasi-static case, where the compression rate is very low, the dissipation will occur during the compression, and no relaxation will be observed (see the dashed lines in Fig. 2a).
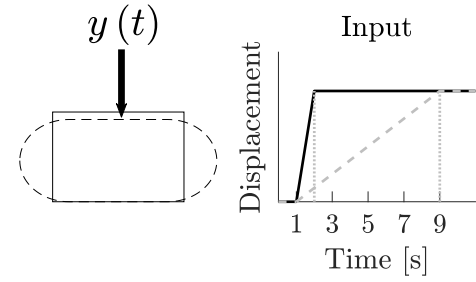

(a) Stress-relaxation experiment
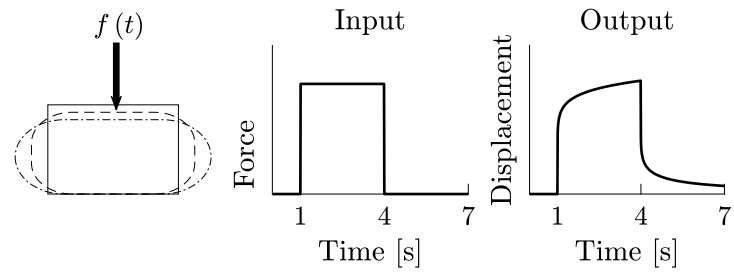

(b) Creep experiment

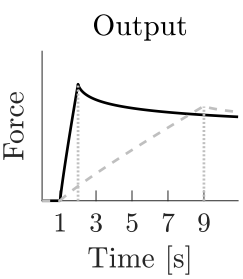

Output
Fig. 2. Mechanical behavior of viscoelastic materials. (a) In stressrelaxation experiments, ramp and hold displacement inputs at various rates (see the middle panel) are applied to the tested materials (see the left panel, where the undeformed and deformed states of a specimen are drawn with solid and dashed lines, respectively). The force responses are analyzed for the magnitude at steady-state and the decay rate (see the right panel). In the middle and right panels, the solid black and dashed grey curves represent the cases for a high and low compression rate, respectively. (b) In creep experiments, step force inputs (see the middle panel) are applied to the tested materials. In the left panel, the undeformed and deformed states of a specimen are drawn with solid, dashed (onset of input) and dash-dotted (offset of input) lines, respectively. The restoration of the material to its undeformed state is observed (see the right panel). The plots are obtained by the simulations of a springpot element for $\alpha=0.1$. 


\subsection{Creep}

Creep can be seen as the dual of stress-relaxation. In a creep experiment, the controlled input is a step force applied for a certain amount of time and released afterward. Initially, we observe an instantaneous deformation like an elastic material. As time progresses, the material further deforms but with an exponentially decaying strain rate. At the instant when the force is released, the initial portion of the strain is recovered, but full recovery takes more time (see Fig. 2b).

\subsection{Phase Difference}

A phase difference occurs between the force and displacement of viscoelastic materials stimulated under a dynamic load (see Fig. 3). The magnitude of this difference can be used to determine the relationship between the energy loss and storage characteristics of a viscoelastic material. The smaller (larger) the phase difference, the more restorative (dissipative) the material is. Therefore, this phase difference is directly related to the perceived bounciness of the material.

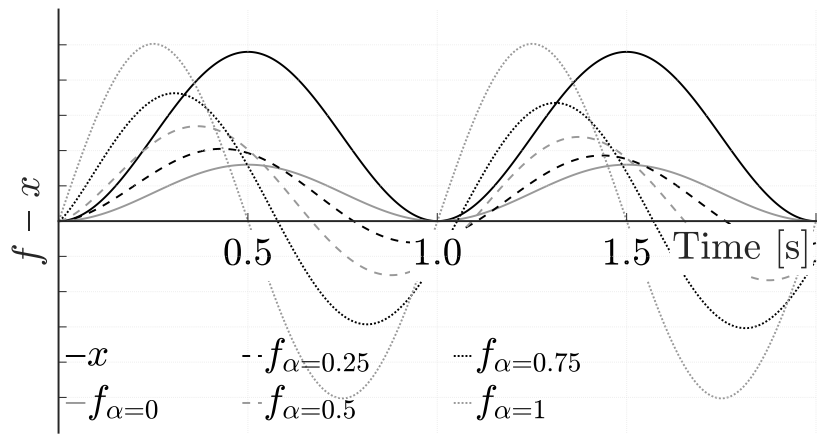

Fig. 3. Phase difference between the displacement $y$ (the black solid line) and force $f$ for different derivative orders $\alpha$. For $\alpha=0$, the $y$ and $f$ (grey solid line) are in phase, and as $\alpha \rightarrow 1$, phase difference increases. Note that for $0<\alpha<1$, the peaks of the sinusoidal curves also decrease over time due to relaxation. For $\alpha=0$, no relaxation occurs; whereas for $\alpha=1$, relaxation is instantaneous.

\subsection{Springpot Element}

Springpot element is the basic building block of fractional viscoelastic models [57]. These models can capture diverse complex material behavior. Regardless, a springpot element can also serve as a representative model reflecting the common viscoelastic material characteristics: stress-relaxation, creep, and phase difference under dynamic loading (see Fig. 2 and 3). Interested readers can find the mathematical formulations of the stress-relaxation and creep characteristics of a springpot element in [58].

The concept of fractional derivative is needed to take full advantage of a springpot element, whose behavior is best analysed in the frequency domain. For a one-dimensional springpot element under a dynamic load of $y(t)=Y e^{i \omega t}$ with magnitude $Y$ and frequency $\omega$, Eq. (1) becomes

$$
f(t)=\chi \frac{d^{\alpha} Y e^{i \omega t}}{d t^{\alpha}}
$$

where $f(t)$ is the force response, and $i$ represents a complex number. Differentiation of the displacement $y(t)$ leads to

$$
f(t)=\chi(i \omega)^{\alpha} Y e^{i \omega t},
$$

Here, one can replace $i^{\alpha}$ with its Euler form:

$$
f(t)=\chi \omega^{\alpha} Y\left(e^{i \pi / 2}\right)^{\alpha} e^{i \omega t} .
$$

or more compactly,

$$
f(t)=\chi \omega^{\alpha} Y e^{i(\omega t+\phi)},
$$

where the phase difference $\phi=\alpha \pi / 2$.

Hence, we can write Eq. (5) in terms of a complex stiffness $k_{C}$ as a function of $\omega$ :

$$
f(t)=k_{C}(\omega) Y e^{i \omega t},
$$

where $k_{C}(\omega)=\left|k_{C}\right| \angle \phi$. So, the relationship between the force response and displacement input can be represented by a magnitude $\left|k_{C}\right|=\chi \omega^{\alpha}$ and phase difference $\phi$. Consequently, we can represent the viscoelastic spectrum of a springpot element in polar coordinates (see Fig. 1). This spectrum is defined from 0 to 1 in terms of $\alpha$. For $\alpha=0$, Eq. (5) reduces to

$$
f(t)=\chi \underbrace{Y e^{i \omega t}}_{y(t)},
$$

which is equivalent to a spring equation, whereas, for $\alpha=1$, the same equation reduces to

$$
f(t)=\chi \underbrace{\omega Y e^{i \omega t+\pi / 2}}_{\dot{y}(t)} .
$$

This is equivalent to a dashpot equation where $\dot{y}(t)$ is the time derivative of the input displacement $y(t)$, i.e., the velocity of the input excitation.

\subsection{Perceptual Terms for Viscoelasticity}

The force-displacement relationship of the springpot element can be described by perceptual terms using the proper choice of adjectives relating to the perceived physical quantities. The springpot model is defined by two parameters: $\chi$ and $\alpha$. Hence, the force response of a springpot element can be described by two perceptual terms relating to the model parameters.

The parameter $\chi$, which scales the force response, is defined as the "firmness" by Scott-Blair and Coppen [49]. The intermediary nature of the word firmness serves the purpose of defining a range between soft and hard materials. According to the online version of the Cambridge Dictionary, the literal definition of firmness is "the quality of not being soft, but not completely hard" [59].

The remaining parameter $\alpha$ determines the angular coordinate of a springpot element on the polar coordinates (see Fig. 1). The physical meaning of the angular coordinate can be interpreted in several different ways: phase difference between input and output, the ratio of energy storage to dissipation capacity, or a transition from being a spring to a dashpot. In either way, this parameter defines a springpot's ability to rebound to its original shape. This quality is described by "springiness" [42], [60], [61] or "bounciness" [60], 
[62]. We avoid the term "springiness" which is derived from spring and sometimes used synonymously with elasticity [62], [63], and instead, choose to use the term "bounciness" in our study.

\section{EXPERIMENT I: Discrimination OF BOUNCI- NESS}

In this experiment, we measured the JND for the bounciness and show how bounciness discrimination performance changes along the angular coordinate of the viscoelastic spectrum.

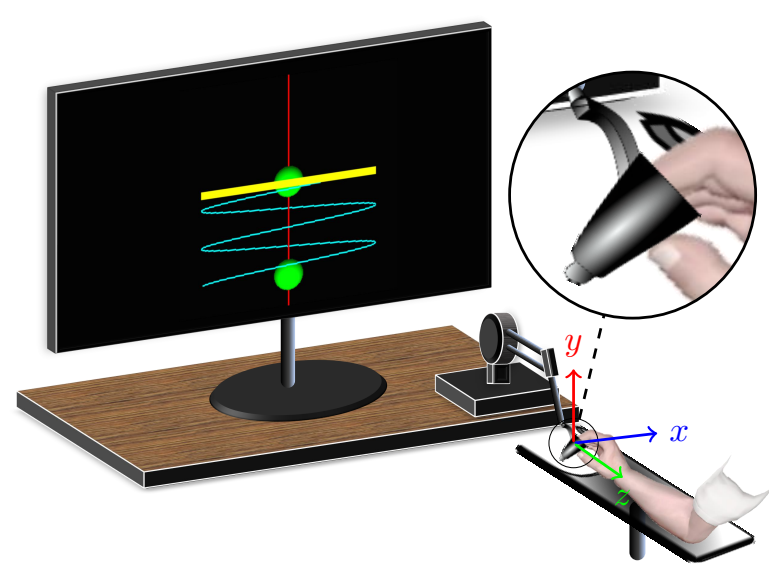

Fig. 4. Experimental setup and the graphical representation of a stimulus. The participants gripped the end-effector of the haptic device in precision grip pose by placing the index finger inside the thimblegimble and supporting it with the thumb (see the magnified part in the circle). The stimulus surface and body are represented by the yellowcolored rectangle and the cyan-colored helix, respectively. Note that in the familiarization phase of the experiment, the surface of the reference and comparison stimuli are color-coded: red for reference and green for comparison (see Section 3.3). Additionally, the movement direction is shown with the red line along the $y$ axis which restricts the participant's movement with a snap constraint, and the green spheres denote the starting and ending positions of the movement.

\subsection{Apparatus}

Visual and haptic stimuli were rendered using a $21.5^{\prime \prime}$ computer screen and a Phantom Premium 1.0 device with the thimble-gimbal attached, respectively (see Fig. 4). To map the workspace of the haptic device to the graphics scene, we used a homogeneous transformation consisting of a pure translation (no rotation). In addition, we projected the graphics scene to the visual display using the orthogonal projection method to avoid any perspective effects.

\subsection{Stimuli}

All stimuli were springpot elements with a force response along the $y$-axis only. The graphical representation of the stimuli consisted of a yellow-colored plate and a cyancolored helical spring under the plate (see Fig. 4). The force response of a stimulus was computed numerically in realtime using our "modified" Grunwald-Letnikov method, as explained in Appendix A.

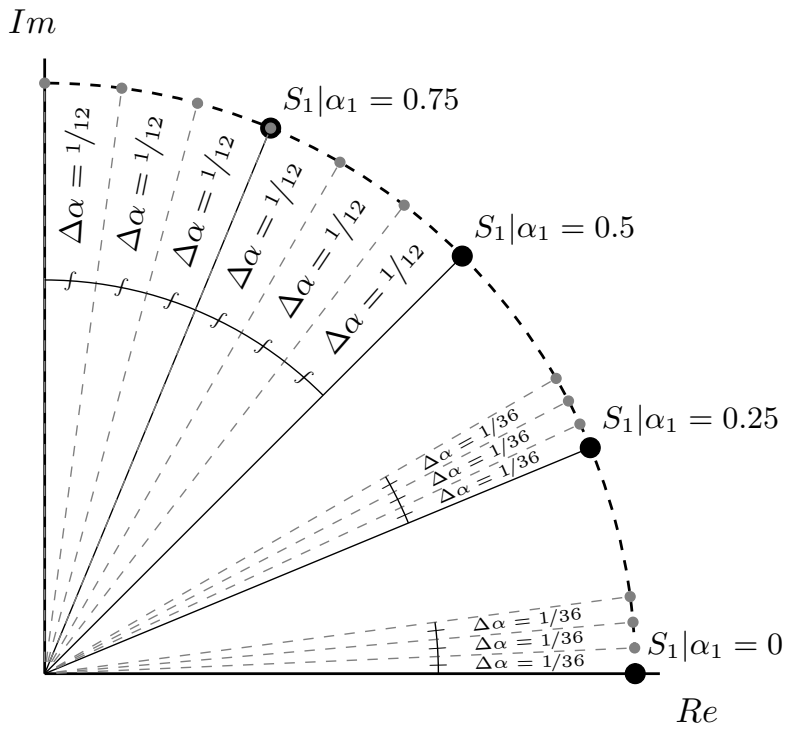

Fig. 5. The viscoelastic spectrum, and the tested ranges for bounciness experiment. The beginning of each range corresponds to a reference stimulus $S_{1}$ (black circle), and three comparison stimuli $S_{2}$ (grey circles) were chosen within each range. The reference and the comparison stimuli differ in the angular coordinates by $\Delta \alpha$. The magnitudes of the force response, i.e., the radial coordinates, were equated according to Eq. (6).

\subsection{Experimental Design}

We designed a signal detection experiment using oneinterval two-alternative forced-choice (1I-2AFC) method. In our experiment, we assumed that the perceived bounciness (i.e., the perception of phase difference) comes from a normally distributed probability density function along the viscoelastic spectrum.

We divided the viscoelastic spectrum of a springpot element into four equal ranges in terms of the phase difference $\phi$ between force response and displacement input. The beginning of each range corresponds to a reference stimulus $S_{1}$ with $\phi=\alpha_{1} \pi / 2$, where the fractional derivative order $\alpha_{1} \in\{0,0.25,0.5,0.75\}$. For each $S_{1}$, we had three comparison stimuli $S_{2}$ with derivative orders $\alpha_{2}$ with equally spaced increments of $\Delta \alpha$, i.e., $\alpha_{2}=\alpha_{1}+\Delta \alpha \times n$, where $n \in\{1,2,3\}$ (see Fig. 5).

In total, there were 12 pairs of stimuli (four references $\times$ three comparisons per reference). Each referencecomparison pair was tested in a different experimental session, and each session was conducted on a different day. This experiment took 12 days to complete for each participant. To randomize the testing order of the four ranges, i.e., reference stimuli, and the three comparison stimuli nested within the ranges, we used the four-by-four Graeco-Latin square design matrix given in Table 2 [64]. Here, the Greek letters denote different ranges: $\beta:=\alpha_{1}=0, \gamma:=\alpha_{1}=0.25$, $\zeta:=\alpha_{2}=0.5$, and $\theta:=\alpha_{1}=0.75$. The Latin letters denote different permutations of the 3 comparison stimuli: $A=\left(\begin{array}{lll}1 & 2 & 3 \\ 1 & 2 & 3\end{array}\right) ; B=\left(\begin{array}{ccc}1 & 2 & 3 \\ 2 & 3 & 1\end{array}\right) ; C=\left(\begin{array}{lll}1 & 2 & 3 \\ 3 & 1 & 2\end{array}\right) ; D=\left(\begin{array}{lll}1 & 2 & 3 \\ 3 & 2 & 1\end{array}\right)$.

\subsection{Experimental Procedure}

Before the beginning of the experiment, the term "bounciness" was explained to the participants, and they were 
TABLE 2

Graeco-Latin Square Design matrix

\begin{tabular}{|c|c|c|c|c|}
\hline & \multicolumn{4}{|c|}{ Participant No. } \\
\hline & 1 & 2 & 3 & 4 \\
\hline \multirow{4}{*}{$\frac{\vec{d}}{0}$} & $\beta A$ & $\gamma D$ & $\zeta B$ & $\theta C$ \\
\hline & $\gamma B$ & $\beta C$ & $\theta A$ & $\zeta D$ \\
\hline & $\zeta C$ & $\theta B$ & $\beta D$ & $\gamma A$ \\
\hline & $\theta D$ & $\zeta A$ & $\gamma C$ & $\beta B$ \\
\hline
\end{tabular}

informed about the nature of the two stimuli in that the reference stimulus $S_{1}$ felt bouncier than the comparison stimulus $S_{2}$. Every session started with a familiarization phase where participants were asked to explore the two stimuli until they were able to differentiate them. During this phase, the participants could select and explore $S_{1}$ (colored as red) or $S_{2}$ (colored as green) without any limitations on the number of explorations per stimulus or the number of switches between the two stimuli. Simultaneous exploration was not possible. Upon completion of the familiarization phase for the first session, the participants were asked whether they could comprehend the difference between the stimuli and felt comfortable with the term "bounciness" to describe the perception of the stimuli. All participants considered "bounciness" as appropriate for describing the stimuli.

The familiarization phase also served as a training for the exploration procedure. While exploring the stimuli, the participants were asked to follow a certain procedure which was the same as that in our previous work [29]. First, the participants came into contact with the stimulus boundary indicated by the yellow plane and waited for the visual cursor at the starting position to change color from red to green before starting the exploration (see Fig. 4). Once the exploration started, a reference cursor appeared. The reference cursor moved along the $y$ axis at a frequency of $\omega=2 \pi \mathrm{rad} / \mathrm{s}$. The participants followed this reference cursor and explored each stimulus for $3 \mathrm{~s}$. During the exploration, the participants always stayed in contact with the stimulus surface, which behaves like a rigid body in that it moves only along the participants' exploration direction and does not deform locally.

We imposed a fixed excitation frequency to attenuate the cues due to the difference in force magnitude $|f| \propto \chi \omega^{\alpha}$ (see 5 ). We adjusted the firmness coefficient $\chi_{2}$ of the comparison stimuli $S_{2}$ with respect to the firmness coefficient $\chi_{1}$ of the reference stimuli $S_{1}$ as:

$$
\chi_{2}=\chi_{1} \frac{\omega^{\alpha_{1}}}{\omega^{\alpha_{2}}},
$$

so that the force magnitudes for $S_{1}$ and $S_{2}$ were equated.

The testing phase started right after the familiarization phase, and each session consisted of 120 trials. In each trial of the testing phase, either $S_{1}$ or $S_{2}$ was randomly presented to the participants with a neutral color (yellow). The participants' task was to explore the stimulus and respond according to the color codes learned in the familiarization phase: red for bouncier and green for otherwise. Halfway through the testing phase, a short break was given to avoid
TABLE 3

Stimulus-Response Matrix

\begin{tabular}{l|l|l|l|}
\multicolumn{2}{c}{} & \multicolumn{2}{c}{ Response } \\
\cline { 3 - 4 } \multicolumn{1}{c|}{} & $R_{1}$ & $R_{2}$ \\
\cline { 2 - 4 } Stimulus & $S_{1}$ & $C R$ (Correct reject) & $F A$ (False alarm) \\
\cline { 2 - 4 } & $S_{2}$ & $M$ (Miss) & $H$ (Hit) \\
\cline { 2 - 4 } & &
\end{tabular}

fatigue. The first 10 trials at the beginning of the two halves of the testing phase were discarded as training trials. All data analyses were performed on the remaining 100 trials.

\subsection{Participants}

Four healthy participants (all engineering graduate students, non-native fluent English speakers, right-handed, one female and three males with an average age of $30.25 \pm$ 8.2 years old) performed the experiments. Each participant spent a total of approximately $6 \mathrm{~h}(12$ conditions $\times 30 \mathrm{~min}$, tested on 12 different days) for the whole experiment. We chose to perform our experiments with well-trained and dedicated subjects for a relatively long testing period. The study was approved by the Koc University Human Ethics Committee, and the participants gave their signed informed consent.

\subsection{Analysis of Perceptual Data}

Based on the participants' responses, we formed two-bytwo response matrices for each reference-comparison pair (see Table 3). Then, we computed the sensitivity index $d^{\prime}$ as

$$
d^{\prime}=z(H)-z(F A),
$$

where $z(\cdot)$ is the inverse of the cumulative distribution function of the standard Gaussian distribution, and $H$ and $F A$ are the hit and false alarm rates obtained from the response matrix, respectively (see Table 3 ). To avoid the $z$ function to take a value of $\pm \infty$, we added an incorrect response to the replies when necessary, i.e., when $H=1$ or $F A=0$.

According to the signal detection theory, for a reference value of $\alpha_{1}$, the ratio $\delta^{\prime}=d^{\prime} / \Delta \alpha$ should have a constant value that depends on the participant's sensitivity to the difference between the reference and comparison stimuli. Thus, for each reference stimulus, the three pairs of $\left(\Delta \alpha \times n \mid n \in\{1,2,3\}, d^{\prime}\right)$ should be on a straight line with the slope $\delta^{\prime}$ and an intercept at 0 . Due to the noise in measurements, one might not get a constant $\delta^{\prime}$, but instead can calculate the mean of the slopes $\bar{\delta}^{\prime}$ using the three $\delta^{\prime}$ values. The reciprocal of the mean slope $\bar{\delta}^{\prime}$, which corresponds to the $\Delta \alpha$ value when $d^{\prime}=1$, is the just-noticeable difference: $\mathrm{JND}=1 / \bar{\delta}^{\prime}$. JNDs estimated as $d^{\prime}=1$, correspond to one standard deviation of the cumulative Gaussian function and thus to $84 \%$ discrimination threshold. Also, the mean JND for the population was found from the mean of the slopes across all participants: JND $=1 /\left(\mu\left(\bar{\delta}^{\prime}\right)\right)$ [24], [65].

\subsection{Analysis of Physical Interaction Data}

We also investigated the relationship between the responses of the participants and several different parameters derived 
from the recorded physical interaction data, i.e., force and displacement signals. Since we use the term bounciness to describe the sensation related to the fractional derivative order $\alpha$, we expected the participants to respond in accordance with the phase difference between the reaction force and displacement. It is also conceivable that, while providing their responses, the participants might have relied on cues other than the phase difference. Previously, Tan et al. used the sensitivity index $d^{\prime}$ as an indicator of the role that mechanical-work cues played when compliance JND was measured with a roving displacement task [7], [24]. They found the $d^{\prime}$ values to be low and variable for some conditions when assuming that the participants were responding to compliance cues. When the same data were reprocessed assuming that the participants were using mechanical-work cues, a higher and most stable $d^{\prime}$ value emerged. This result was taken as evidence that the participants may have relied more on mechanical-work cues than compliance cues when performing compliance discrimination [24].

In this work, we adopted the approach in [24] due to the similar experimental methods. We estimated the average (physical) distances $\hat{d}^{\prime}$ between the reference and comparison stimuli for several candidate parameters and then normalized these values with respect to the corresponding (perceptual) $d^{\prime}$ values. For clarity, we used the term "sensitivity index" with the perceptual data and "normalized distance" with physical parameters even though they were computed in similar ways.

The physical interaction parameters were chosen based on our viscoelastic model and also the earlier studies where similar analyses were performed [10], [11], [24]. The selected parameters were categorized into frequency-domain parameters:

- $\quad k_{S}$ : Real (storage related) part of complex stiffness

- $k_{L}$ : Imaginary (loss related) part of complex stiffness

- $\quad \eta$ : Loss factor $\left(k_{S}\right) /\left(k_{L}\right)$

- $b_{L}$ : Imaginary part of force over the magnitude of velocity (Imaginary part of the mechanical impedance)

- $\phi$ : Phase difference between force and displacement,

and time-domain parameters:

- $W$ : Positive part of the mechanical-work done by the participant

- $f_{\max }$ : Maximum of the force peaks

- $\bar{f}$ : Mean of the force peaks

- $y_{\max }$ : Maximum of the displacement peaks

- $\bar{y}$ : : Mean of the displacement peaks

- $v_{\max }$ : Maximum of the velocity peaks

- $\bar{v}$ : Mean of the velocity peaks

- $\quad k_{I}$ : Initial local stiffness (first force peak divided by the corresponding indentation depth)

- $\quad k_{f i t}$ : Slope of the zero-intercept line fitted to force as a function of displacement (force over position, FOP, as in [10])

- $b_{f i t}$ : Slope of the zero-intercept line fitted to force as a function of velocity [10].

The frequency-domain parameters were computed based on the most dominant frequency components of the relevant data. The frequency components of the data and their magnitudes were identified using the Discrete Fourier
Transform (DFT) functionality of Matlab. Following the DFT, we adjusted the phase of each signal using a rotation matrix that took the displacement data as the reference, i.e., the transformed displacement data always had a phase of $0 \mathrm{rad}$.

Some of the selected physical interaction parameters depend on the programmed material properties of the stimuli and the motion of the participants who were instructed to follow a visual cursor. Since our exploration procedure was prescribed, one might think that the values of these parameters can be pre-computed and then the normalized distances can be easily computed using the methods from the previous section. However, the participants' performance in following the visual cursor determines the actual values of the interaction parameters derived from the recorded force, displacement, and velocity data. So, the physical interaction parameters do not match the expected ones perfectly. Because of these deviations or the parameter's nature, the recorded physical interaction data do not necessarily form clearly distinct clusters. Therefore, we cannot use the methods described in the previous section to compute normalized distances based on the physical interaction data.

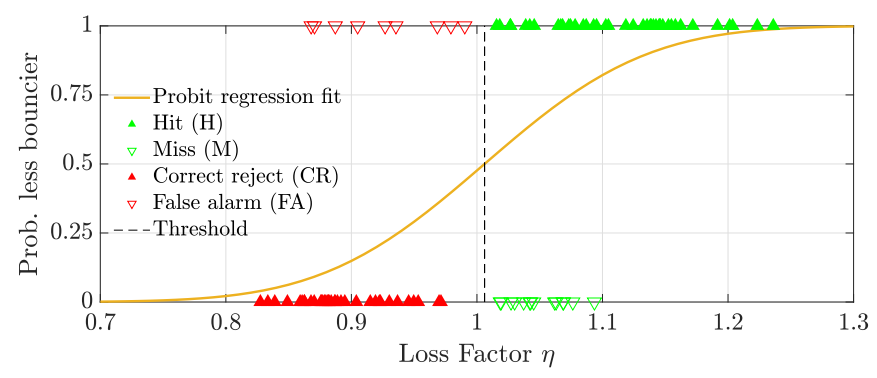

(a) Normal cumulative function fit to data

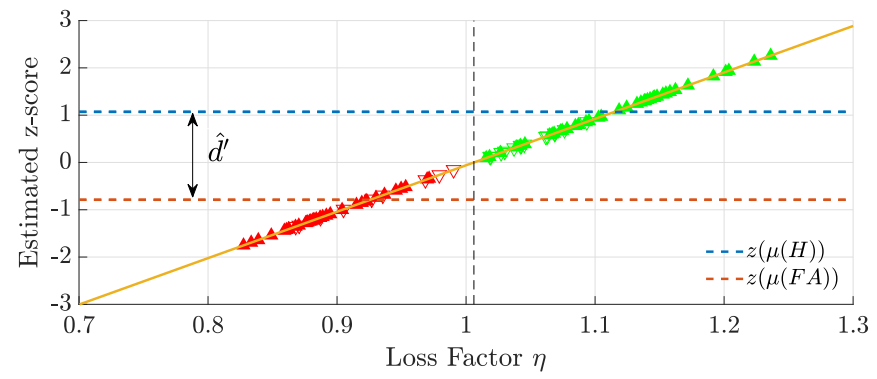

(b) Data mapped to the fitted line

Fig. 6. An example showing the curve fit to loss factor data versus participant responses using probit function. (a) The triangles denote the data points, i.e., the loss factor data of the trials vs the participant responses (1: Less bouncy, 0: Bouncier). (b) The data points are mapped to the fitted line as z scores. Based on the fit, the green data points are classified as less bouncy stimuli and the red ones as the bouncier.

To investigate the relation between the physical data and the responses of the participants, we fitted generalized linear models (GLM) using a Bernoulli distribution and a probit link function (see Fig. 6a). The fitted linear models transform the participants' responses ( 0 or 1$)$ to $z$-scores as functions of physical interaction parameters (see Fig. 6b). Using these linear models, we classified the stimuli experienced by the participants as noise or signal depending on the sign of the estimated $\mathrm{z}$-scores. If the estimated $\mathrm{z}$ score of a trial is positive (negative), then this trial is classified as a signal (noise). Then, we can estimate a parameter specific 
normalized distance $\hat{d}^{\prime}$ between the means of the physical parameters in $z$ coordinates.

\subsection{Results}

The slopes $\bar{\delta}^{\prime}$ and their reciprocals (JNDs) are plotted in Fig. $7 \mathrm{a}$ and Fig. $7 \mathrm{~b}$, respectively. A linear relationship was observed between the mean JND values of the whole group and the reference $\alpha_{1}>0$, and a linear regression model was fitted using Matlab (see Fig. 7b). The fitted line has a slope of $0.1452(t(1)=20.0065, p=0.0318)$ and an intercept of $-0.0012(t(1)=-0.3088, p=0.8093)$ with $R^{2}=0.998$.

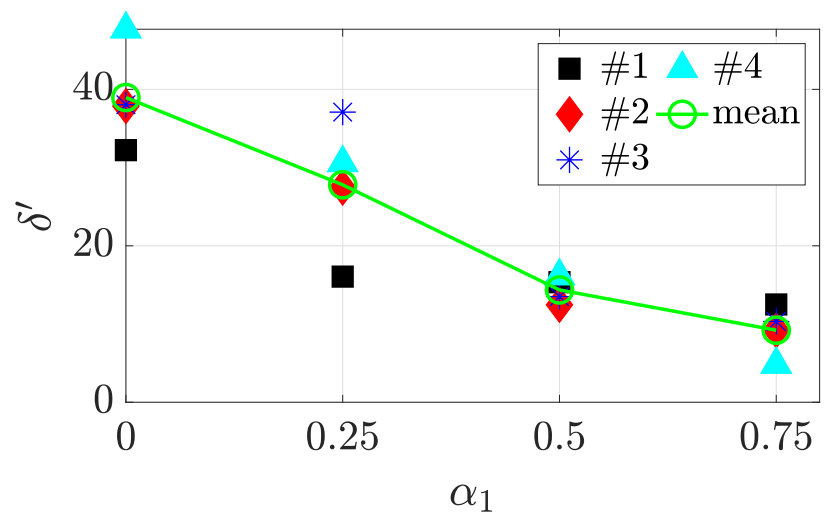

(a) Slopes $\delta^{\prime}$

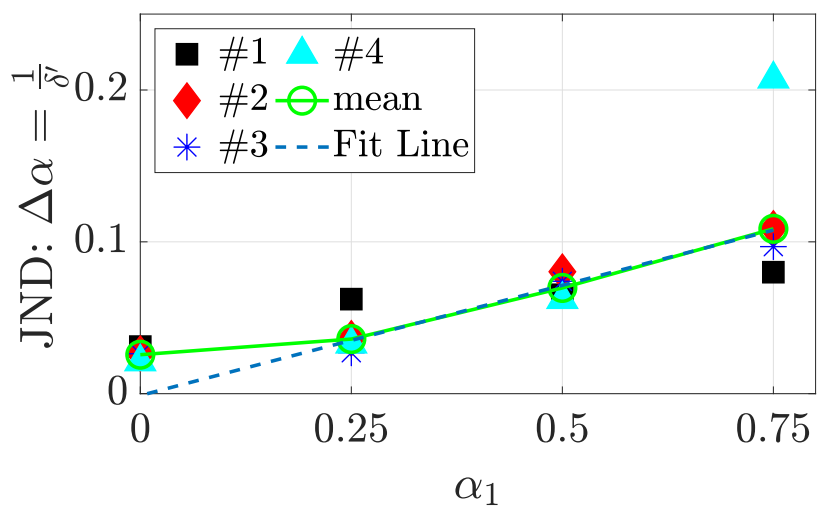

(b) JNDs

Fig. 7. The slope (a) and JND (b) results of each participant and the group means for all references. In $7 \mathrm{~b}$, a blue dashed line is fitted to the mean JNDs of references for $\alpha_{1}>$.0

The above results were computed with respect to changes in the derivative order $\alpha$ which results in a change in phase difference $\phi$. Nevertheless, the participants might have based their decisions on the differences in other physical parameters arising from the interaction with the stimuli. To investigate the possible role of these parameters in their perception, we computed the ratio of normalized distance over the sensitivity index $\left(\hat{d}^{\prime} / d^{\prime}\right)$ for several physical interaction parameters and averaged the resulting values across the increments and the participants for all references. In Fig. 8, for each reference, we report the three parameters having the largest ratios between signal and noise.

We observed that imaginary part of the mechanical impedance $b_{L}$ was among the top three parameters for all references. Also, the ratio for the phase difference $\phi$ was at least 0.97 for all references. In contrast, for $\alpha=0.75$, mechanical-work $W$ had the highest ratio.

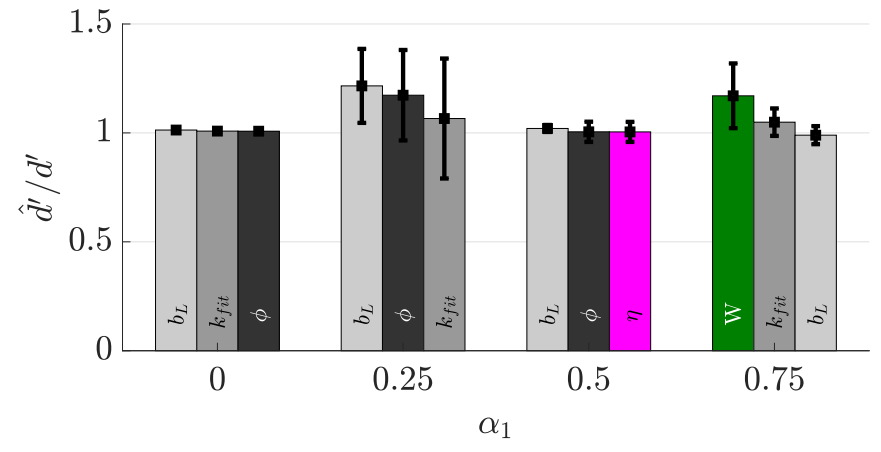

Fig. 8. The ratios of estimated $\hat{d}^{\prime}$ of the physical parameters to $d^{\prime}$ in Experiment $\mathrm{I}$. The results are the mean values averaged over the participants and the $3 \Delta \alpha$ increments, and the errorbars show the standard errors of the means. For each reference, only the three parameters with the largest ratios are reported.

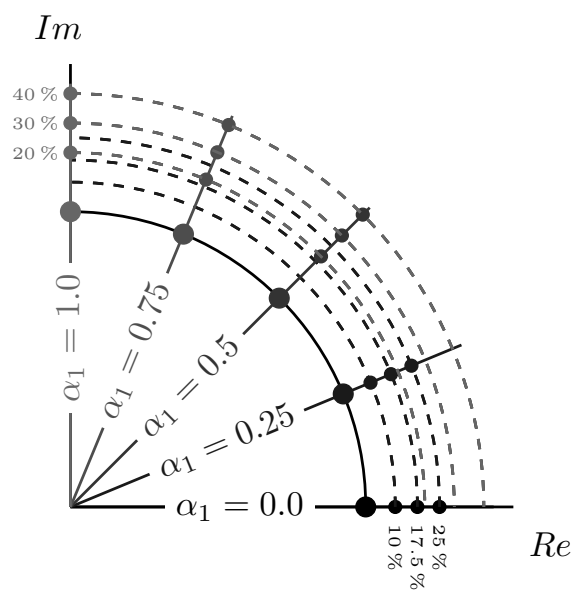

Fig. 9. The viscoelastic spectrum, and the tested ranges for firmness experiment. The beginning of each range corresponds to a reference stimulus $S_{1}$ (large circles), and three comparison stimuli $S_{2}$ (smaller circles) are chosen within each range. The comparison stimuli have the same $\alpha$ values as their respective reference stimuli. The reference and the comparison stimuli differ in the radial coordinates by $\Delta \chi$. The levels of $\Delta \chi$ are shown with the grey arcs.

\section{EXPERIMENT II: DISCRIMINATION OF FIRMNESS}

Another perceptual characteristic of a springpot element is its firmness. Assuming that bounciness perception does not change for a fixed value of $\alpha$, we can characterize firmness solely by $\chi$. In this experiment, we measured the JNDs for the firmness of springpot elements that have a fixed location along the viscoelastic spectrum in terms of phase difference, i.e., the different reference stimuli were characterized by their $\alpha$ values while the comparison stimuli differed from the references only in $\chi$ (see Fig. 9). With this experiment, we aimed to fill the gap in the literature between the stiffness (firmness when $\alpha=0$ ) and damping/viscosity (firmness when $\alpha=1$ ) JND values.

\subsection{Methods}

We utilized the same apparatus, experimental design, and data analysis methods as in the bounciness experiment. However, one major difference was in the exploration procedure. In the current case, a change in $\chi$ does not affect the angular coordinate of a springpot element along the 
viscoelastic spectrum. Therefore, unlike in the previous case, we do not have to impose a specific movement frequency on the participants to attenuate extra cues. This freedom results in a visuo-haptic interaction that is much closer to our daily life experience.

Another difference is the inclusion of $\alpha=1$, which increased the number of reference stimuli $S_{1}$ to five with the derivative orders $\alpha_{1} \in\{0,0.25,0.5,0.75,1\}$. We again had three comparison stimuli $S_{2}$ with firmness coefficients $\chi_{2}=\chi_{1}+\Delta \chi$ (see Fig. 9).

The firmness coefficient of the reference stimuli was normalized as

$$
\chi_{1}=\chi_{0} \frac{\omega^{\alpha_{0}}}{\omega^{\alpha_{1}}}=\frac{\chi_{0}}{\omega^{\alpha_{1}}},
$$

where $\omega=2 \pi \mathrm{rad} / \mathrm{s}$ was chosen as a reference excitation frequency, $\alpha_{0}=0, \chi_{0}=0.3 \mathrm{~N} / \mathrm{mm}$, and the unit of $\chi_{1}$ is $\mathrm{N} \cdot \mathrm{s}^{\alpha_{1}} / \mathrm{mm}$. This normalization was performed for achieving comparable force responses across the reference stimuli.

The above changes increased the number of pairs (testing conditions) to 15 ( 5 references $\times 3$ comparisons). Consequently, the total experimentation time increased to approximately $7.5 \mathrm{~h}(15$ conditions $\times 30 \mathrm{~min})$ per participant. The size of the Graeco-Latin Square design matrix is increased to $5 \times 5$ to accommodate the increase in the number of references. Therefore, we needed to have an equal number of references, permutations of increments, and participants. So, we included an additional permutation $E=\left(\begin{array}{lll}1 & 2 & 3 \\ 2 & 1 & 3\end{array}\right)$ and recruited five new participants (all engineering graduate students, right handed, and males with an average age of $32.2 \pm 5$ years old).

\subsection{Results}

The slopes of the lines fitted to the $d^{\prime}$ and the JND values as a percentage of the reference $\chi_{1}$ values, i.e., Weber fractions WFs, are depicted in Fig. 10a and 10b, respectively. The slopes and hence the JND values do not follow a clear trend with respect to $\alpha_{1}$ unlike in the first experiment. A repeated measures ANOVA in SPSS showed no significant effect of $\alpha_{1}$ $(F(4,16), p=0.3981)$, i.e., level of bounciness on firmness WF. Also, no significant change has been observed for the pairwise comparisons with respect to $\alpha_{1}$ levels (all $p>0.5$ ).

As in the previous experiment, we computed the ratios of the estimated $\hat{d}^{\prime}$ of the chosen physical interaction parameters to the $d^{\prime}$ of $\Delta \chi$ and averaged these ratios across participants. In Fig. 11, the averaged ratios of the physical parameters with the three largest values are reported. According to this plot, frequency-domain parameters $\left(k_{S}\right.$ for $\alpha=0,0.25$ and 0.5 , and $b_{L}$ for $\alpha=0.75$ and 1$)$, and regression parameters $\left(k_{f i t}\right.$ for $\alpha=0,0.25$ and 0.5 ; and $b_{\text {fit }}$ for $\alpha=0.5,0.75$ and 1) appeared to have the largest ratios between signal and noise stimuli.

\section{Discussion AND Conclusion}

In this study, we have considered viscoelasticity as a mechanical response displaying a spectrum of behaviors varying from pure elastic (spring) to pure viscous (damper). We also adopted the "bounciness" and "firmness" from

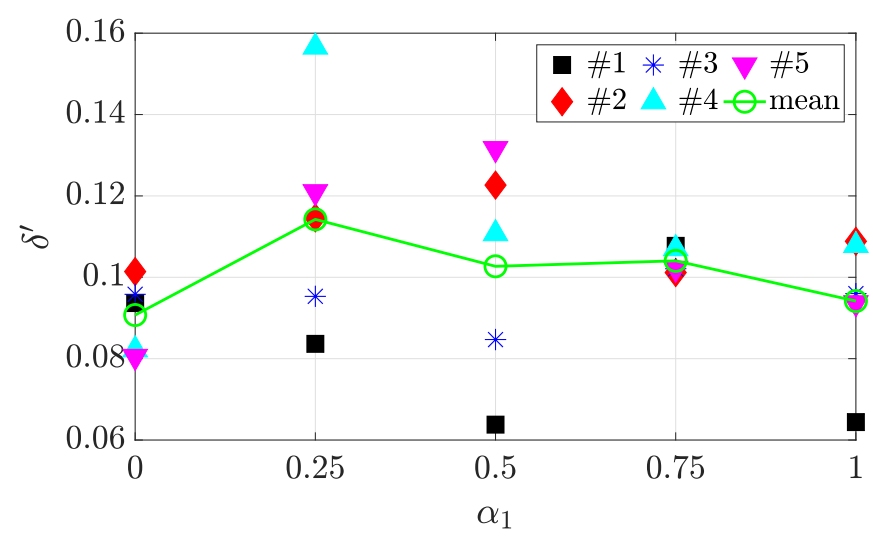

(a) Slopes $\delta^{\prime}$

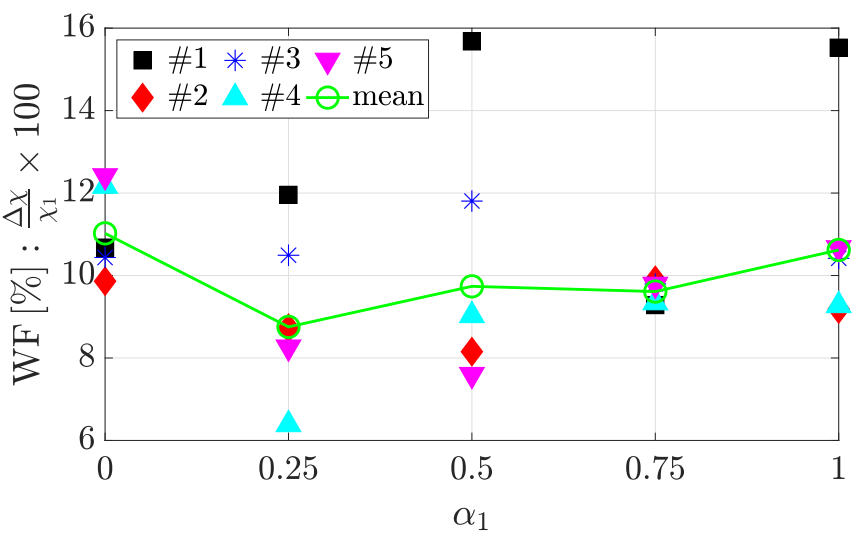

(b) JNDs in Weber fractions

Fig. 10. (a) The slope and (b) JND in Weber fractions results of each participant and the group means for all references.

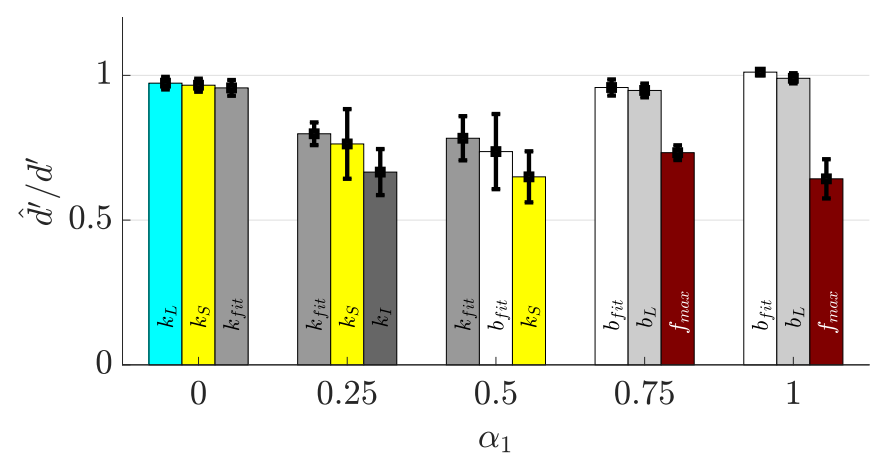

Fig. 11. The ratios of estimated $\hat{d}^{\prime}$ of the physical parameters to $d^{\prime}$ in Experiment II. The results are the mean values averaged over the participants, and the errorbars show the standard errors of the means. For each reference, only the three parameters with the largest ratios are reported.

the psychorheology field as the perceptual terms describing the sensations associated with viscoelastic materials. At various locations on the viscoelastic spectrum, we measured the bounciness and firmness JNDs via two psychophysical experiments.

\subsection{Bounciness Experiments}

In the first experiment, bounciness JND was found to be monotonically increasing with the $\alpha$ parameter of the springpot element. For references with $\alpha_{1}$ values larger than 
zero, the mean JND values followed a linear trend with its intercept being approximately zero (see Fig. 7b). This trend is reminiscent of Weber's law which states that the JND of a physical quantity is a constant fraction of the reference value. We should also note that transforming our modeling-basis might result in a change in the measured "normalized distances" between the stimuli categorized as signal and noise. If the participants made their decisions based on a specific cue, the estimated normalized distance for this cue should be larger than the other estimated distances. This argument acknowledges the fact that although the experimenter has clear definitions of signal and noise based on the model, the participants might have their own definitions based on the sensory cues they rely on. The ratio of the normalized distance to the sensitivity index $\left(\hat{d}^{\prime} / d^{\prime}\right)$ tells more about this argument (see Fig. 8). A ratio lower than one $\left(d^{\prime}>\hat{d}^{\prime}\right)$ suggests that the parameter manipulated by the experimenter ( $\alpha$ in bounciness and $\chi$ in firmness experiments) is more effective than the physical parameter in question on the participants' perception. Analysis of this ratio revealed that the frequency-domain parameters were the ones most likely affecting bounciness perception for $\alpha_{1}<0.75$. In this regard, the imaginary part of the mechanical impedance $b_{L}$ was always in the top three parameters. Except for the last reference, the phase difference $\phi$ was also among the top three parameters, and its ratio was at least 0.97 for all references. However, for the last reference, mechanical-work $W$ had the highest ratio (see Fig. 8 for $\left.\alpha_{1}=0.75\right)$.

Based on these findings, we can explain the increase in JND with respect to $\alpha$ by the energy dissipation characteristics of the stimuli which is related to the imaginary part of the mechanical impedance $b_{L}$. In the case of a pure spring, the addition of even some dissipation was easily detected by the participants. However, as $\alpha$ grows, both the reference and comparison stimuli became more dissipative, and the participants might have had difficulty identifying the relatively more dissipative stimulus. Instead, they might have relied on the cues related to effort, i.e., mechanicalwork $W$.

\subsection{Firmness Experiments}

In the firmness experiment, we did not observe a significant change, let alone an increase in the JND in Weber fractions WFs as the stimuli ranged from a pure spring $(\alpha=0)$ to a pure damper $(\alpha=1)$. Our results show that the firmness WFs does not change when the phase difference is fixed across the stimuli. Nevertheless, this does not necessarily mean that firmness discrimination of viscoelastic stimuli is independent of its viscous component. In fact, a delay in force feedback (a phase difference in force response with respect to displacement input) has already been shown to affect both the perceived stiffness (firmness in our context) and discrimination performance [10], [13], [35]. Nonetheless, as discussed in [66], the WFs reported in earlier studies for viscosity (13.6\% [25]; $34 \%$ [27]) are larger than the WFs reported for elasticity (8\% [24]; $23 \%$ [26]).

In terms of the experimental methods, our study is most similar to [24] and [25] where signal detection experiments were performed with the elastic and viscous stimuli ren- dered by an electromechanical device. In those studies, compliance and viscosity WFs were $\sim 8 \%$ and $\sim 14 \%$, respectively. Nevertheless, the WF values reported in [25] have a high standard deviation among participants. Later in [67], Beauregard and Srinivasan acknowledged this high standard deviation and attributed it to the relatively poor discrimination performance of one of the three participants. Furthermore, they included new data from another viscosity JND experiment with different reference stimuli varying in the damping coefficient for a fixed displacement of $30 \mathrm{~mm}$, and the new results showed the viscosity WF to be below $10 \%$. So, the viscosity JND reported as Weber fractions in [25] seemed to be overestimated.

We can also compare our results with other haptic perception studies on viscosity JND. In [68], the authors investigated the masking effect of an additional stiffness and inertia on the viscosity JND using a programmable electromechanical device. According to the results of the control condition where no masking was applied, the mean WF was reported as being around $10 \%$. In contrast, in earlier studies where real liquid test specimens were used, the mean WF values were reported as $30 \%$ in [49] and above $30 \%$ in [9]. However, their results have high intercondition and inter-participant variations, and the WFs of some participants are considerably below $10 \%$ for some conditions. These large variations also support our choice of using a relatively small number of well-trained participants.

To understand the cues used for discrimination of firmness, we computed the normalized distances based on the parameters obtained from the physical interaction data. We observed that the ratio $\left(\hat{d}^{\prime} / d^{\prime}\right)$ was never greater than one (see Fig. 11). This result suggests that the participants took the frequency variations in their movements into account when they responded. Therefore, the manipulated parameter $\chi$ provided the relevant cues for the perception of firmness.

\subsection{Effect of (Presence of) Visual and (Lack of) Tactile Feedback}

Although our experimental methods are similar to those used in [24], [25] and [67], two major differences were present in our experiments: (1) visual feedback was displayed to the participants, and (2) the indentation distance was not fixed. Nevertheless, our JND results in Weber fractions are comparable to those reported in [24] and [67]. While the uncontrolled displacement was expected to yield a lower discrimination performance similar to the roving displacement condition (22\% [7]), the inclusion of the visual cues might have aided the participants in our experiments. It is conceivable that our participants compensated for uncontrolled displacement with the help of visual cues. Earlier studies have already shown that reliability in haptic stiffness estimation can be improved with the integration of visual information [12], [69], [70]. Regardless of the sensory integration process, both Wu et al. [21] and Varadharajan et al. [22] reported statistically significant improvement in stiffness JND when visual feedback was available with kinesthetic force feedback.

On the contrary, visual feedback was found not to improve the sensitivity to the difference between reference 
and comparison stimuli for real specimens [23]. The authors argued that this result was due to the higher reliability of local surface deformation cues in firmness estimation. Real specimens enable a natural interaction and provide valuable cutaneous information which is limited in the case of electromechanical (EM) devices displaying kinesthetic force feedback. The implications of the reduced cutaneous feedback have been studied by using elastic stimulus with a rigid plate attached to its top surface [8], [71]. In [71], Srinivasan and LaMotte showed that participants could discriminate pairs of rubber stimuli much better than pairs of spring cells with rigid plates on their top surfaces. Bergmann Tiest and Kappers measured the softness (firmness) JNDs using rubber stimuli with and without rigid plates attached to the top and bottom surfaces. They reported an increase in Weber fraction from $15 \%$ to $50 \%$ when the rigid surfaces were used, i.e., the cutaneous cues were reduced.

On the other hand, real specimens are usually prepared by curing silicone rubber mixtures [8], [15], [17], [36], [72], and it is known that silicone rubber exhibits nonlinear and viscoelastic material behavior [73]. Hence, care should be given to their mechanical characterization. Nevertheless, so far, mechanical characterization of these specimens for the psychophysical investigation of haptic perception has been nonstandard, and the recent work by Gerling et al. is an important step towards solving this problem [74]. More specifically, tuning the viscoelastic properties of a silicone rubber while controlling its material nonlinearities is a challenge that has not been solved yet.

Conversely, our work required simultaneous tuning of viscoelastic material properties related to firmness and bounciness. For this reason, we have chosen to use an EM device in the current work. Despite the reduced cutaneous cues, EM devices can still successfully simulate the scenarios involving tool-object interactions. In that respect, our results, for example, could be useful in the design and evaluation of surgical simulators and teleoperated surgical systems where simplifications in tissue models and reductions in data transfer rates are required to meet real-time constraints.

\subsection{Conclusion}

In summary, we observed that the JND values for bounciness increased as a function of the fractional derivative order, whereas the WF for firmness was almost constant across the five equally spaced points along the angular coordinate. When bounciness and firmness are considered as the terms for assessing the perception of viscoelastic materials, we can successfully explain the participants' decision-making using frequency-domain parameters. The frequency-domain parameters themselves provide cues related to the energy storage and dissipation characteristics of the stimuli. Energy storage and loss characteristics relate to a sense of effort which might have been used as an intuitive indicator for the perception of viscoelastic stimuli.

In our future studies, we will explore the viscoelastic spectrum as a bivariate domain, and investigate the effect of interaction between the parameters $\chi$ and $\alpha$ on the perceived bounciness and firmness. We also plan to investigate the roles temporal combination and integration of different sensory signals play in firmness perception.

\section{APPENDIX A \\ NUMERICAL IMPLEMENTATION OF Fractional DeRIVATIVE}

Although there exists several different definitions of fractional derivatives, the Grunwald-Letnikov derivative is the most popular one for numerical implementations. Grunwald-Letnikov derivative of an arbitrary function of time $g(t)$ is

$$
{ }_{a} D_{t}^{\alpha} g(t) \approx \frac{1}{\Delta t^{\alpha}} \sum_{j=0}^{\lfloor(t-a) /(\Delta t)\rfloor} w_{j}^{(\alpha)} g(t-j \Delta t),
$$

where

$$
w_{j}^{(\alpha)}=(-1)^{\alpha}\left(\begin{array}{l}
\alpha \\
j
\end{array}\right),
$$

and $\lfloor$.$\rfloor is the floor operator. This fractional derivative of$ order $\alpha$ is defined from time $a$ to time $t$ with a discrete time step of $\Delta t$. A close inspection of Eq. (8) reveals that the fractional derivatives depend on the entire history of the function of interest. As time progresses, this method becomes both computationally and memory-wise intensive. Since haptic rendering requires real-time force updates at a rate of $1 \mathrm{kHz}$, the computation of fractional order derivatives with the standard Grunwald-Letnikov derivative can cause problems for our purposes.

A common method to remedy this problem is the shortterm memory (STM) principle [75]. According to STM principle, one can set a limit to the number of data points from the history of the differentiated function, and rely on the most recent data which have higher weights. However, we observed that stress-relaxation behavior was not captured well using this approach. Instead, we chose to use a limited number of data points by successive downsampling during the computation of the fractional order derivatives. With the number of data points limited to $N$, the discrete sampling rate at the $k^{t h}$ iteration was reduced to $\lceil k / N\rceil$ instead of performing the computation at the haptic update rate. Our approach not only captures the stress-relaxation behavior better but also lowpass filters the numerical derivative, which results in a smoother derivative function (see Fig. 12).

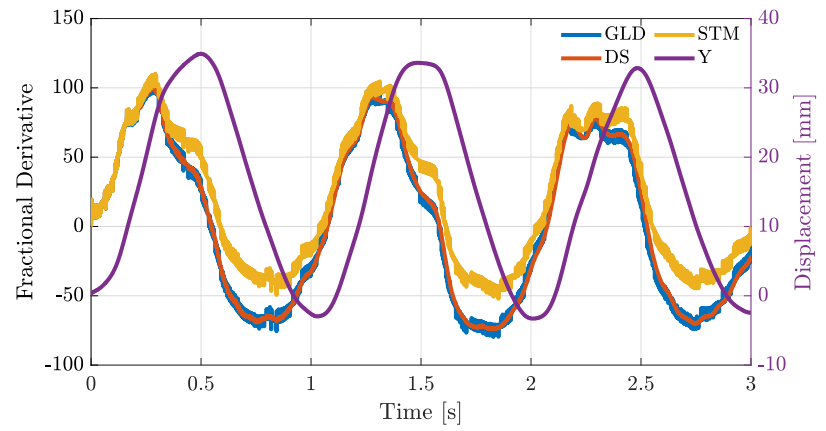

Fig. 12. The standard Grunwald-Letnikov derivative (GLD) with 3000 data points, downsampled (DS) version with 100 data points, and shortterm memory (STM) version with 100 data points. The input displacement $(\mathrm{Y})$ is also shown in purple color with its axis label given on the right. 


\section{ACKNOWLEDGMENTS}

Ozan Caldiran is funded by the BIDEB-2211 Student Fellowship Program of The Scientific and Technological Research Council of Turkey (TUBITAK). The first author thanks Yusuf Aydin for the introduction of fractional derivative, and Omer Sirin and Utku Erdem for their feedback during the design of the experiments.

\section{References}

[1] Y. C. Fung, Biomechanics : Mechanical Properties of Living Tissues. New York: Springer, 1993. ISBN 9781441931047

[2] A. Sassoon, J. Riehl, A. Rich, J. Langford, G. Haidukewych, G. Pearl, and K. J. Koval, "Muscle viability revisited: Are we removing normal muscle? A critical evaluation of dogmatic debridement level," Journal of Orthopedic Trauma, vol. 30, pp. 17-21, 2016.

[3] C. Basdogan, M. Sedef, M. Harders, and S. Wesarg, "VR-based simulators for training in minimally invasive surgery," IEEE Computer Graphics and Applications, vol. 27, no. 2, pp. 54-66, 2007.

[4] P. Hinterseer, S. Hirche, S. Chaudhuri, S. Member, E. Steinbach, and M. Buss, "Perception-based data reduction and transmission of haptic data in telepresence and teleaction systems," IEEE Transactions on Signal Processing, vol. 56, no. 2, pp. 588 - 597, 2008.

[5] J. Kammerl, I. Vittorias, V. Nitsch, B. Faerber, E. Steinbach, and S. Hirche, "Perception-based data reduction for haptic forcefeedback signals using velocity-adaptive deadbands." Presence: Teleoperators \& Virtual Environments, vol. 19, no. 5, pp. $450-462$, 2010.

[6] R. L. Klatzky, D. Pawluk, and A. Peer, "Haptic perception of material properties and implications for applications," Proceedings of the IEEE, vol. 101, no. 9, pp. 2081-2092, 2013.

[7] H. Z. Tan, N. I. Durlach, G. L. Beauregard, and M. A. Srinivasan, "Manual discrimination of compliance using active pinch grasp: The roles of force and work cues," Perception $\mathcal{E}$ Psychophysics, vol. 57, no. 4, pp. 495-510, 1995.

[8] W. M. Bergmann Tiest and A. M. L. Kappers, "Cues for haptic perception of compliance," IEEE Transactions on Haptics, vol. 2, no. 4, pp. 189-199, 2009.

[9] W. M. Bergmann Tiest, A. C. L. Vrijling, and A. M. L. Kappers, "Haptic discrimination and matching of viscosity," IEEE Transactions on Haptics, vol. 6, no. 1, pp. 24-34, 2013.

[10] I. Nisky, F. A. Mussa-Ivaldi, and A. Karniel, "A regression and boundary-crossing-based model for the perception of delayed stiffness," IEEE Transactions on Haptics, vol. 1, no. 2, pp. 73-82, 2008.

[11] F. E. Van Beek, J. F. Dennis, H. Nijmeijer, W. M. Bergmann Tiest, and A. M. L. Kappers, "The effect of damping on the perception of hardness," in IEEE World Haptics Conference, 2015, pp. 82-87.

[12] M. Kuschel, M. Di Luca, M. Buss, and R. L. Klatzky, "Combination and integration in the perception of visual-haptic compliance information," IEEE Transactions on Haptics, vol. 3, no. 4, pp. 234244, 2010.

[13] A. Pressman, L. J. Welty, A. Karniel, and F. A. Mussa-Ivaldi, "Perception of delayed stiffness," The International Journal of Robotics Research, vol. 2612, no. 11, pp. 1191-1203, 2007.

[14] M. Rank, Z. Shi, H. J. Müller, and S. Hirche, "Perception of delay in haptic telepresence systems," Presence, vol. 19, no. 5, pp. 389-399, 2010.

[15] R. H. LaMotte, "Softness discrimination with a tool," Journal of Neurophysiology, vol. 83, no. 4, pp. 1777-1786, 2000.

[16] U. Koçak, K. L. Palmerius, C. Forsell, A. Ynnerman, and M. Cooper, "Analysis of the jnd of stiffness in three modes of comparison," in Haptic and Audio Interaction Design, 2011, pp. 2231.

[17] E. Fakhoury, P. Culmer, and B. Henson, "The effect of vision on discrimination of compliance using a tool," International Journal of Human-Computer Interaction, vol. 30, no. 11, pp. 882-890, 2014.

[18] F. Barbagli, K. Salisbury, C. Ho, C. Spence, and H. Z. Tan, "Haptic discrimination of force direction and the influence of visual information," ACM Transactions on Applied Perception, vol. 3, no. 2, pp. 125-135, 2006.

[19] F. E. Van Beek, W. M. Tiest, and A. M. Kappers, "Anisotropy in the haptic perception of force direction and magnitude," IEEE Transactions on Haptics, vol. 6, no. 4, pp. 399-407, 2013.
[20] L. Kaim and K. Drewing, "Exploratory strategies in haptic softness discrimination are tuned to achieve high levels of task performance," IEEE Transactions on Haptics, vol. 4, no. 4, pp. 242-252, 2011.

[21] W.-C. Wu, C. Basdogan, and M. A. Srinivasan, "Visual, haptic, and bimodal perception of size and stiffness in virtual environments," in Proceedings of the American Society of Mechanical Engineers Dynamic Systems and Control Division, vol. 67, 1999, pp. 19-26.

[22] V. Varadharajan, R. Klatzky, B. Unger, R. Swendsen, and R. Hollis, "Haptic rendering and psychophysical evaluation of a virtual three-dimensional helical spring," in Symposium on Haptics Interfaces for Virtual Environment and Teleoperator Systems 2008 Proceedings, Haptics, 2008, pp. 57-64.

[23] C. Cellini, L. Kaim, and K. Drewing, "Visual and haptic integration in the estimation of softness of deformable objects," i-Perception, vol. 4, no. 8, pp. 516-531, 2013.

[24] H. Z. Tan, X. D. Pang, and N. I. Durlach, "Manual resolution of length, force, and compliance," in Proceedings of ASME Winter Annual Meeting: Advances in Robotics, vol. 42, 1992, pp. 13-18.

[25] G. L. Beauregard, M. A. Srinivasan, and N. I. Durlach, "The manual resolution of viscosity and mass," ASME Dynamic Systems and Control Division, vol. 1, pp. 657-662, 1995.

[26] L. A. Jones and I. W. Hunter, "A perceptual analysis of stiffness," Experimental Brain Research, vol. 79, pp. 150-156, 1990.

[27] L. A. Jones and I. W. Hunter, "A perceptual analysis of viscosity," Experimental Brain Research, vol. 94, no. 2, pp. 343-351, 1993.

[28] I. Nisky, A. Pressman, C. M. Pugh, F. A. Mussa-Ivaldi, and A. Karniel, "Perception and action in teleoperated needle insertion," IEEE Transactions on Haptics, vol. 4, no. 3, pp. 155-166, 2011.

[29] O. Caldiran, H. Z. Tan, and C. Basdogan, "An investigation of haptic perception of viscoelastic materials in the frequency domain," in IEEE Haptics Symposium, 2018, pp. 222-228.

[30] A. Israr, Y. Li, V. Patoglu, and M. K. O'Malley, "Passive and active discrimination of natural frequency of virtual dynamic systems," IEEE Transactions on Haptics, vol. 2, no. 1, pp. 40-51, Jan 2009. doi: 10.1109/TOH.2008.21

[31] W. Fu, A. Landman, M. M. van Paassen, and M. Mulder, "Modeling human difference threshold in perceiving mechanical properties from force," IEEE Transactions on HumanMachine Systems, vol. 48, no. 4, pp. 359-368, Aug 2018. doi: 10.1109/THMS.2018.2844212

[32] W. Fu, M. M. van Paassen, D. A. Abbink, and M. Mulder, "Framework for human haptic perception with delayed force feedback," IEEE Transactions on Human-Machine Systems, pp. 1-12, 2018. doi: 10.1109/THMS.2018.2885401

[33] S. J. Lederman and R. L. Klatzky, "Hand movements: A window into haptic object recognition," Cognitive Psychology, vol. 19, pp. 342-368, 1987.

[34] N. Macfadyen, C. G. Maher, and R. Adams, "Number of sampling movements and manual stiffness judgments." Journal Of Manipulative And Physiological Therapeutics, vol. 21, no. 9, pp. 604-610, 1998.

[35] M. Di Luca, B. Knörlein, M. O. Ernst, and M. Harders, "Effects of visualhaptic asynchronies and loadingunloading movements on compliance perception," Brain Research Bulletin, vol. 85, pp. 245$259,2011$.

[36] K. Drewing, Exploratory Movement Strategies in Softness Perception. London: Springer London, 2014, pp. 109-125.

[37] C. M. Pugh, "Application of national testing standards to simulation-based assessments of clinical palpation skills," MILITARY MEDICINE, vol. 178, p. 55, 2013. doi: 10.7205/MILMED-D$13-00215$

[38] J. Konstantinova, G. Cotugno, P. Dasgupta, K. Althoefer, and T. Nanayakkara, "Palpation force modulation strategies to identify hard regions in soft tissue organs," PLoS ONE, vol. 12, no. 2, 2017.

[39] S. Rogosin and F. Mainardi, "George William Scott Blair - the pioneer of factional calculus in rheology," Communications in Applied and Industrial Mathematics, vol. 6, no. 1, pp. 1-20, 2014.

[40] G. W. S. Blair, "Psychorheology: Links between the past and the present," Journal of Texture Studies, vol. 5, no. 1, pp. 3-12, 1974.

[41] D. W. Hadley and J. D. Weber, "Rheologieal nomenelature," Rheollogica Acta, vol. 14, pp. 1098-1109, 1975.

[42] R. Jowitt, "The terminology of food texture," Journal of Texture Studies, vol. 5, no. 3, pp. 351-358, 1974.

[43] A. S. Szczesniak, "Classification of textural characteristics," Journal of Food Science, vol. 28, no. 4, pp. 385-389, 1963. 
[44] G. V. Civille and A. S. Szczesniak, "Guidelines to training a texture profile panel," Journal of Texture Studies, vol. 4, pp. 204-223, 1973

[45] H. T. Lawless and H. Heymann, Sensory Evaluation of Food, 2010.

[46] R. J. Marshall, "Combined instrumental and sensory measurement of the role of fat in food texture," Food Quality and Preference, vol. 2, pp. 117-124, 1990.

[47] J. Benedito, R. Gonzalez, C. Rosselló, and A. Mulet, "Instrumental and expert assessment of mahon cheese texture," Journal of Food Science, vol. 65, no. 7, 2000 .

[48] T. Morávková and P. Stern, "Rheological and textural properties of cosmetic emulsions," Applied Rheology, vol. 21, no. 3, pp. 1-6, 2011.

[49] G. W. S. Blair and F. M. V. Coppen, "The subjective judgment of the elastic and plastic properties of soft bodies; the differential thresholds for viscosities and compression moduli," in Proceedings of the Royal Society B: Biological Sciences, vol. 128, no. 850, 1939, pp. 109-125.

[50] G. W. S. Blair and F. M. V. Coppen, "The subjective conception of the firmness of soft materials," The American Journal of Psychology, vol. 55, no. 2, pp. 215-229, 1942.

[51] G. W. S. Blair and F. M. V. Coppen, "The estimation of firmness in soft materials," The American Journal of Psychology, vol. 56, no. 2, pp. 234-246, 1943.

[52] G. W. S. Blair, B. C. Veinoglou, and J. E. Caffyn, "Limitations of the newtonian time scale in relation to non-equilibrium rheological states and a theory of quasi-properties," in Proceedings of the Royal Society of London. Series A. Mathematical and Physical Sciences, 1947.

[53] G. W. S. Blair, "The subjective assessment of the consistency of materials in relation to physical measurements," Journal of Society of Cosmetic Chemists, vol. 17, pp. 45-56, 1966.

[54] M. Ayyildiz, G. Aktas, and C. Basdogan, "Effect of solution and post-mortem time on mechanical and histological properties of liver during cold preservation," Biorheology, vol. 51, pp. 47-70, 2014.

[55] O. Tokatli and V. Patoglu, "Generalized virtual environment models for haptic rendering," in Workshop on Haptic Teleoperation, $\operatorname{TrC}$ IFToMM Symposium on Theory of Machines and Mechanisms, 2015.

[56] O. Tokatli, "Fractional order control in haptics," Ph.D. dissertation, Sabanci University, 2015.

[57] H. Schiessel and A. Blumen, "Hierarchical analogues to fractional relaxation equations," Journal of Physics A: Mathematical and General, vol. 26, no. 19, pp. 5057-5069, oct 1993.

[58] F. F. Mainardi, Fractional calculus and waves in linear viscoelasticity : An introduction to mathematical models. Imperial College Press, 2010.

[59] (2018) Meaning of "firmness" in the English dictionary. Cambridge Advanced Learner's Dictionary \& Thesaurus. [Online]. Available: https://dictionary.cambridge.org/dictionary/english/firmness

[60] A. S. Szczesniak, "Texture is a sensory property," Food Quality and Preference, vol. 13, no. 4, pp. 215-225, 2002.

[61] T. Faber, A. Jaishankar, and G. McKinley, "Describing the firmness, springiness and rubberiness of food gels using fractional calculus. part i: Theoretical framework," Food Hydrocolloids, vol. 62, pp. 311324, 2017.

[62] M. C. Bourne, Food Texture and Viscosity: Concept and Measurement, 2002.

[63] J. G. Davis, "The rheology of cheese, butter and the rheology of milk products," Journal of Dairy Research, vol. 8, no. 2, pp. 245-264, 1937.

[64] M. Cavazzuti, "Design of experiments," in Optimization Methods. Berlin, Heidelberg: Springer Berlin Heidelberg, 2013, pp. 13-42.

[65] L. A. Jones and H. Z. Tan, "Application of psychophysical techniques to haptic research," IEEE Transactions on Haptics, vol. 6, no. 3, pp. 268-284, 2013.

[66] I. Nisky, P. Baraduc, and A. Karniel, "Proximodistal gradient in the perception of delayed stiffness," Journal of Neurophysiology, vol. 103, pp. 3017-3026, 2010.

[67] G. L. Beauregard and M. A. Srinivasan, "Sensorimotor interactions in the haptic perception of virtual objects," MIT Touch Lab, Tech. Rep., 1997.

[68] M. Rank, T. Schauß, A. Peer, S. Hirche, and R. L. Klatzky, "Masking effects for damping JND," in Haptics: Perception, Devices, Mobility, and Communication, 2012, pp. 145-150.

[69] R. L. Klatzky and B. Wu, Visual-Haptic Compliance Perception. London: Springer London, 2014, pp. 17-30.

[70] M. Di Luca and M. O. Ernst, Computational Aspects of Softness Perception. London: Springer London, 2014, pp. 85-106.
[71] M. A. Srinivasan and R. H. LaMotte, "Tactual discrimination of softness," Journal of Neurophysiology, vol. 73, no. 1, pp. 88-101, 1995.

[72] A. Metzger, A. Lezkan, and K. Drewing, "Integration of serial sensory information in haptic perception of softness." Journal of Experimental Psychology: Human Perception and Performance, vol. 44, no. 4, pp. 551-565, 2018.

[73] J. J. M. De Bever, "Dynamic behaviour of rubber and rubberlike materials," TU Eindhoven, Eindhoven, Tech. Rep., 1992.

[74] G. J. Gerling, S. C. Hauser, B. R. Soltis, A. K. Bowen, K. D. Fanta, and Y. Wang, "A standard methodology to characterize the intrinsic material properties of compliant test stimuli," IEEE Transactions on Haptics, 2018. doi: 10.1109/TOH.2018.2825396

[75] D. Baleanu, Fractional Calculus: Models And Numerical Methods, ser. Complexity, Nonlinearity, and Chaos. World Scientific, 2012, no. 3 .

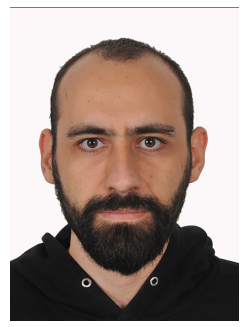

Ozan Caldiran is a Ph.D. candidate and a TUBITAK BIDEB fellow at Koc University, Istanbul, Turkey. He received his BSc degree in Mechatronics Engineering from Sabanci University in 20009 and his MSc degree in Robotics, Systems, and Control from ETH Zurich in 2011. During his Master studies, he worked at German Aerospace Center (DLR) as an intern and also completed his thesis there. He is a student member of the IEEE.

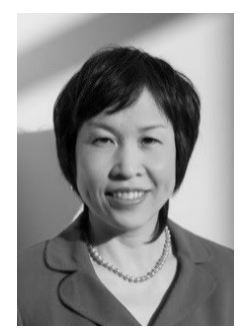

Hong Z. Tan received her Bachelor's degree in Biomedical Engineering from Shanghai Jiao Tong University in 1986, and earned her Master and Doctorate degrees (in 1988 and 1996 respectively), both in Electrical Engineering and Computer Science, from the Massachusetts Institute of Technology. She is currently a professor of electrical and computer engineering, mechanical engineering (by courtesy) and psychological sciences (by courtesy) at Purdue University. She has served as an associate editor of the IEEE Transactions on Haptics from 2007 to 2012 and since 2016, and received a meritorious service award in 2012. She is a Fellow of the IEEE.

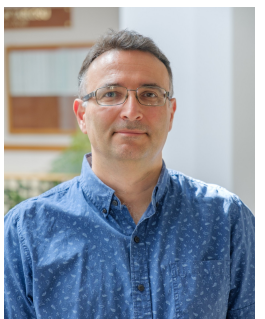

Cagatay Basdogan received the Ph.D. degree in mechanical engineering from Southern Methodist University, in 1994. He is a faculty member in the mechanical engineering and computational sciences and engineering programs at Koc University, Istanbul, Turkey. $\mathrm{He}$ is also the director of the Robotics and Mechatronics Laboratory, Koc University. Before joining Koc University, he worked at NASAJPL/Caltech, MIT, and Northwestern University Research Park. His research interests include haptic interfaces, robotics, mechatronics, biomechanics, medical simulation, computer graphics, and multi-modal virtual environments. $\mathrm{He}$ is currently the associate editor in chief of the IEEE Transactions on Haptics and serves on the editorial boards of the IEEE Transactions on Mechatronics, Presence: Teleoperators and Virtual Environments, and Computer Animation and Virtual Worlds journals. He is a senior member of the IEEE. In addition to serving in programme and organizational committees of several haptics conferences, he chaired the IEEE World Haptics Conference in 2011. 